\title{
La construcción de una "nueva" mujer argelina: diario de una guerrillera
}

\author{
For the construction of a "new" Algerian woman: diary of a \\ maquisarde
}

\author{
Julieta Chinchilla \\ Universidad de Buenos Aires (Argentina) \\ julietachinchilla@gmail.com
}

\begin{abstract}
Resumen
Este artículo busca analizar cómo desde la columna Journal d'une maquisarde ("Diario de una guerrillera") -que apareció en el diario "El Moudjahid" durante los meses de junio y agosto de 1959, órgano oficial del FLN argelino-, se buscó recrear la imagen de una "nueva" mujer argelina. El hecho de tomar un texto fundacional elaborado en un contexto de guerra, nos permitirá reflexionar sobre la construcción de nuevas subjetividades en el corto plazo, ya sea para equilibrar tensiones y ansiedades generadas por la presencia de mujeres en la guerra, como para ir sentando las bases que sustentarían el rol de las mujeres luego de la independencia.
\end{abstract}

\section{Palabras Clave}

Argelia; Mujeres; Guerra; Nacionalismo; Género.

\begin{abstract}
This article seeks to analyse the construction of a "new" Algerian woman based on the column analysis Journal d'une maquisarde ("Diary of a maquisarde"), that appeared between June and August 1959 in the newspaper "El Moudjahid" (FLN's official publication). The decision a foundational text, which was written in the context of a war, will allow us to reflect on the construction of a new female subjectivity in the short term, either to balance tensions and anxieties generated by the presence of women in the war, or to imagine what might sustain the role of women after independence.
\end{abstract}

\section{Keywords}

Algeria; Women; War; Nationalism; Gender. 
La guerra de independencia de Argelia (1954-1962) fue uno de los conflictos más emblemáticos del período de la descolonización de Asia y África en la segunda mitad del siglo XX. Las denuncias de tortura y desaparición forzada de personas por parte de Francia, fueron parte de la campaña internacional que hizo conocida la lucha del Frente de Liberación Nacional (FLN) argelino por todo el mundo. Sin embargo, la presencia de mujeres en las filas del FLN fue algo que también llamó la atención, y fue aprovechado por el movimiento nacionalista para ganar simpatías a nivel internacional. En los años de guerra, las militantes argelinas fueron conocidas por su participación en la "Batalla de Argel”, a través de los escritos de Frantz Fanon que fueron traducidos en varios idiomas-, y por las denuncias de tortura que alcanzaron relevancia internacional, como en los casos de Djamila Bouhired y Djamila Boupacha.

Ahora, ¿cómo afectó la participación de las mujeres en relación al rol que tenían las argelinas antes del estallido de la guerra? ¿Cómo se transformó la idea de "mujer" y "feminidad" durante los años de guerra? Existen varios trabajos que buscaron responder estas preguntas desde diversos puntos de análisis (Amrane-Minne, 1994; Sambron, 2007; MacMaster, 2009; Chinchilla, 2014; Vince, 2015), sin embargo, en este trabajo nos centraremos en estudiar particularmente cómo se fueron operando cambios en torno a la imagen de la mujer argelina a partir del análisis de la columna "Journal d'une maquisarde", publicada durante los meses de junio y agosto de 1959 por el periódico "El Moudjahid", órgano oficial del FLN argelino¹.

La elección de esta columna se dio por considerarla un texto fundacional, ya que es la primera vez que el FLN le dio espacio a una mujer para que relate en primera persona su experiencia en la guerra -además de la historia de sus otras compañeras, permitiendo construir de esta manera un retrato oficial de la "nueva" mujer argelina. Por este motivo, es que se aprovecha un texto fundacional para dar cuenta de la construcción de una nueva subjetividad femenina a partir de un discurso oficial en un contexto de guerra.

El hecho de que la columna sea un testimonio de guerra redactado en pleno conflicto, permite analizar los cambios en los sistemas de valores que se dan en estas coyunturas, convirtiéndose dicho testimonio en un terreno privilegiado para los estudios de género. Por esta razón, en el corto plazo del acontecimiento se pueden notar las tensiones surgidas en la coyuntura del conflicto, a diferencia de una

\footnotetext{
1 Para el análisis de la columna, se trabajó con la edición francesa de "El Moudjahid", impresa en Yugoslavia durante la guerra de independencia argelina. Esta versión no es idéntica a la impresa en Túnez. La versión yugoslava fue "adaptada" para ganar la simpatía de la opinión pública internacional y la columna que busca analizar el presente artículo debe entenderse como un aporte importante para lograr este objetivo. La edición de "El Moudjahid" en árabe es -según el historiador Gilbert Meyner- "más izquierdista y más secular en comparación El Moudjahid [en francés], ya que los autores no sentían la necesidad constante de demostrar los orígenes árabo-islámicos de Argelia" (Meynier G. 2002).
} 


\section{La construcción de una "nueva” mujer argelina: diario de una guerrillera}

perspectiva de la larga duración, en donde los conflictos bélicos aportarían en un proceso de democratización de las mujeres.

A partir de lo expuesto, nos centraremos en el análisis de la coyuntura del conflicto para poder delinear las nuevas características que deberían obtener las argelinas en un nuevo país independiente. Como podremos analizar, este intento no se hará sin tensiones ni ambigüedades propias de las ansiedades que provocaron los cambios en una sociedad en plena transformación.

\section{El concepto de "nueva" mujer en el discurso nacionalista del Norte de África y Medio Oriente}

Tras la colonización de las regiones del Norte de África y Medio Oriente -por parte de las principales potencias europeas a lo largo del siglo XIX y principios del siglo XX-, la religión se convirtió en una de las principales trincheras desde donde se quería preservar la identidad cultural de dichas sociedades colonizadas, siendo la mujer la principal guardiana de estos valores. En el caso argelino en particular, el hecho de que la duración y la escala de la colonización hayan sido mucho más agudas ${ }^{2}$, llevó a que la mujer argelina adquiriera una importancia simbólica aún más profunda en lo que se refiere al resguardo de la identidad. Esta situación podría explicar el por qué de las ideas del reformista egipcio Qasim Amin³ (1863-1908) sobre la necesidad de educar a una "nueva" mujer para la nación, no permearon entre los reformistas argelinos -a pesar que las mismas hayan circulado por Argelia a inicios del siglo XX-, como había sucedido en otros países de la región. De hecho, no existe ningún equivalente a este intelectual en territorio argelino.

En las primeras décadas del siglo XX, las ideas de Amin fueron continuadas por otros intelectuales árabes, como el tunecino Tahar Haddad (1899-1935). Sin embargo, en Argelia fueron combatidas por uno de los principales referentes de la Nahda argelina, el fundador de la Asociación de Ulemas ${ }^{4}$. Ben Badis tildaba a las críticas que Haddad tenía sobre el uso del velo, el matrimonio arreglado, la poligamia y la repudiación como "anti-islámicas" y occidentalistas. En este contexto, no es difícil concluir que el debate en torno al rol de la mujer argelina estaba caracterizado por una visión binaria, que por un lado esencializaba a las "mujeres musulmanas", y por

\footnotetext{
${ }^{2}$ La conquista colonial de Argelia por parte de Francia comenzó en 1830 cuando, en términos generales, la era del imperialismo colonial en Asia y África comenzó a fines del siglo XIX y principios del siglo XX. Por otro lado, Argelia no fue "una simple colonia", sino un territorio de poblamiento blanco y jurídicamente fue considerada como departamento francés, es decir, en términos formales no era reconocida como una jurisdicción por fuera del hexágono.

${ }^{3}$ Qasim Amin (1863-1908) fue un intelectual y jurista egipcio. Es considerado uno de los principales referentes de la corriente del modernismo islámico o Nahda, además de ser reconocido como "el primer feminista" del mundo árabe, ya que desarrolló toda su obra en torno al rol de la mujer en la nueva sociedad árabe.

${ }^{4}$ La Asociación de Ulemas fue fundada por el reformista musulmán Ben Badis (1889-1940) en 1931. Esta asociación fue una de las corrientes nacionalistas que más influencia tuvo a mediados del siglo XX. Su lema era: “Una religión, el islam; una lengua, el árabe; una Nación, Argelia”.
} 


\section{Julieta Chinchilla}

otro lado señalaba a la "cultura francesa" como algo contrario y ajeno a la sociedad argelina 5 . Este binarismo también era utilizado por el discurso colonialista francés, pero en un sentido inverso, ya que lo utilizaba para señalar el "oscurantismo" de la sociedad argelina a partir del lugar que ocupaban las mujeres y así justificar su política colonial sobre el territorio.

En el contexto de la guerra de independencia argelina, el debate sobre el rol de la mujer se convirtió en un punto central en la lucha para ganar "los corazones y mentes" de la población argelina (Sambron 2007, MacMaster 2009). El FLN surgió con el inicio de la guerra de independencia en noviembre del año 1954. Este frente estuvo compuesto por todas las tendencias del nacionalismo argelino, desde las más conservadoras hasta las más radicales. Sin embargo, fueron las corrientes más progresistas las que fueron ganando importancia en la conducción del movimiento, además de encargarse de la política internacional y de las relaciones exteriores (Byrne, 2016). A este respecto, el surgimiento de un nuevo nacionalismo radical que conducía la guerra, estuvo influido por los discursos de modernidad y desarrollo característicos de esos años. Por este motivo, no es de extrañar que el FLN haya impulsado un discurso sobre la mujer que no sólo buscaba expresar la sociedad moderna e igualitaria a la que aspiraba, sino que también pretendía ganarse a la opinión pública internacional, actor clave para el reconocimiento del derecho a la autodeterminación del pueblo argelino en la Organización de Naciones Unidas.

Con esta lógica, no es de extrañar que una buena parte del discurso del Frente de Liberación Nacional argelino insistía en la idea de que la liberación del dominio colonial era una precondición para la liberación de la mujer argelina. De hecho, el presidente del Gobierno Provisional de la Revolución Argelina (GPRA, por sus siglas en francés), Ferhat Abbas, había sido uno de los pocos nacionalistas influenciados por las ideas de Qasim Amin desde la década del 1940.

\section{La nueva mujer argelina}

Según la académica Kumari Jayawardena (2016), todos los movimientos nacionalistas del Tercer Mundo basaron su discurso de género en la igualdad de las mujeres en el sistema legal, en la eliminación de prácticas claramente discriminatorias, el derecho al voto y la educación extendida. Sin embargo, estos discursos nunca abordaron la subordinación de las mujeres en el ámbito familiar. Esta tensión es la que vamos a poder constatar a lo largo de la columna que queremos analizar.

\footnotetext{
${ }^{5}$ Este debate es comparable al planteo de Kumari Jayawardena (2016) -y otras intelectuales que han estudiado el desarrollo del feminismo en el Tercer Mundo-, cuando menciona que muchas veces el feminismo en el Tercer Mundo fue tomado como una ideología de la burguesía local alienada, que llevaba a la pérdida de la identidad cultural, $y$, por lo tanto, a las responsabilidades familiares de las mujeres locales.
} 


\section{La construcción de una "nueva” mujer argelina: diario de una guerrillera}

"Journal d'une maquisarde", es un testimonio escrito por una mujer que presenta todas las características que señala el historiador Luc Capdevila (2011) para referirse a las memorias de mujeres en guerra: participación voluntaria de las mujeres en un espacio que a priori se les estaba prohibido; no hay retórica de sufrimiento y victimización, sino que existe una exaltación de la acción, además de alegría y orgullo por participar de la guerra; y se señala un constante deseo por servir a la causa. Finalmente, y es interesante señalar, que como otros testimonios de mujeres de la época (década de 1950), no se hace un análisis desde una perspectiva feminista de la participación de las mujeres.

"Journal..." es una columna anónima que apareció con regularidad en el diario El Moudjahid entre junio y agosto de 1959. La autora dice ser una joven mujer de clase media urbana que dejó sus estudios para participar del conflicto ${ }^{6}$. Si bien estas características no son las que representan a la mayoría de las mujeres que participaron de la guerra de independencia en Argelia (Amrane-Minne, 1994), la maquisarde o guerrillera, plantea la necesidad de testimoniar por todas las mujeres que combatieron.

La cronología de la participación de las mujeres en la guerra que se reconstruye a través de la columna, se centra en los años en los que su presencia femenina fue más significativa: desde la huelga estudiantil de mayo de 1956, hasta su retirada a las fronteras (ante la creciente represión de las fuerzas coloniales francesas) en 1959. Sin embargo, más allá de analizar esta columna desde una secuencia de sucesos, resulta interesante analizarla como una genealogía que busca describir el nacimiento y las características de esta nueva mujer argelina.

Desde esta perspectiva, resulta llamativo que este testimonio "bajo supervisión"7 haya privilegiado rescatar la figura de una joven de clase media urbana -así se presenta quién escribe la columna- en detrimento de una mujer del ámbito rural. El discurso oficial del FLN siempre privilegió la lucha que se desarrollaba en los espacios rurales, por sobre la lucha urbana, siendo el modelo ideal de combatiente, aquel de la región de las montañas de los Aurès. Por este motivo, al momento de querer visibilizar la participación femenina, es interesante notar que se privilegia a

\footnotetext{
${ }^{6}$ Aunque no se sabe con exactitud quién escribió la columna, algunos testimonios afirman que la verdadera autora de la columna fue la reconocida escritora argelina, Assia Djebar. También otros autores mencionan que si bien es difícil determinar la autoría de la columna, sí es seguro que Djebar haya sido su editora. Estas afirmaciones son verosímiles si tomamos en cuenta que Assia Djebar fue redactora de "El Moudjahid" en Túnez. Fort Pierre-Louis, Chaulet-Achour Christiane (2013), La France et l'Algérie en 1962 - de l'histoire aux représentations textuelles d'une fin de guerre, París: Karthala, p.106; Chaulet-Achour Christiane (2012), «Écrits algériennes et guerre d'indépendance. Témoignages et créations » en Confluences Méditarranée, N 81, p. 189 a 203, p. 192 ; Cheurfi Achour (2004), Écrivains algériens: dictionnaire biographique, Alger : Casbah, p. 147 ; Déjeux Jean (1994), La littérature féminine de langue française au Maghreb, París : Karthala, p.160, 191

7 Todos los artículos publicados por El Moudjahid en estos años eran discutidos previamente por un comité político editorial. AAVV (2011). El Moudjahid. Un journal de combat (1956-1962). Rouiba: Éditions ANEP.
} 
una joven del ámbito urbano en detrimento de las ciento de miles de mujeres que colaboraban con el maquis en las zonas rurales. ${ }^{8}$

Esta elección estaría en sintonía con lo desarrollado por lxs historiadores Nel MacMaster (2009) y Natalya Vince (2015), cuando en su trabajo afirman que fueron las militantes urbanas las protagonistas como sujetos y agentes de propaganda. Para ejemplificar esta afirmación, Vince, rescata los casos de las tres icónicas militantes llamadas "Djamila" (Bouhired, Bouazza y Boupacha), y de cómo sus casos fueron conocidos en la escena internacional a partir de la publicación de artículos y libros impulsados por comités de solidaridad con el FLN, en donde se denunciaban las injusticias y represión del colonialismo francés a partir de los abusos cometidos contra estas tres militantes 9 . Por su parte, el libro "La mort de mes frères", escrito desde la cárcel por una de las pocas dirigentes mujeres del FLN de la zona autónoma de Argel, Zohra Drif, también es rescatado por la autora, ya que presenta todos los estereotipos clásicos del discurso nacionalista: la participación y el compromiso de las mujeres siempre aparece relacionado en cuanto a su estatus de esposas, madres, hermanas (Vince, 2015).

Ahora, aunque coincidimos con la autora sobre el hecho de que las mujeres del ámbito rural permanecieron ajenas a los discursos y propaganda de género nacionalista, consideramos que aún falta explicar el por qué fueron las mujeres de clase media urbana las elegidas para ser protagonistas y agentes de dicha propaganda.

Según nuestro punto de vista, el hecho de elegir a mujeres jóvenes, urbanas y educadas para la propaganda de género del FLN, se realizó de una manera consciente y con una intencionalidad política. Por un lado, la utilización de la imagen de mujeres jóvenes y "modernas" participando en las filas del FLN tenía como objetivo contrarrestar todo un paquete de medidas que había dispuesto el gobierno colonial francés para la "emancipación de las mujeres" en el año 1959, como parte de su estrategia en la guerra psicológica. ${ }^{10}$ Por otro lado, el objetivo de la dirección

\footnotetext{
${ }^{8} \mathrm{~A}$ pesar que en el imaginario colectivo se recuerda a las militantes del FLN como mujeres jóvenes que participaron en la guerrilla, según el registro del Ministerio de ex Combatientes de Argelia, de las 10.469 mujeres registradas, el 19\% se incorporó en la estructura del Ejército de Liberación Nacional (ALN por sus siglas en francés), el resto fueron militantes civiles. Un poco menos de la mitad del total de militantes registradas era menor de 30 años, y el 78\% entre ellas pertenecía el medio rural. TALEB IBRAHIMI, K. (2012). “Les Algeriénnes et la guerre de libération nationale. L'émergence des femmes dans l'espace public et politique au cours de la guerre et l'après-guerre", en HARBI, M. y STORA, B. La guerre d'Algérie. Millau: Pluriel.

${ }_{9}^{9}$ Estas tres militantes urbanas fueron arrestadas y torturadas, para luego ser condenadas a muerte al ser acusadas de poner bombas en diferentes bares de la ciudad de Argel. Además de numerosos artículos publicados en diferentes periódicos del mundo, vale destacar los libros "Pour Djamila" de los abogados Georges Arnaud y Jacques Vergès y "Djamila Boupacha" de Giselle Halimi y Simone Beauvoir. También el caso de Djamila Bouhired buscó ganar la simpatía del mundo árabe a través del film egipcio, dirigido por Youssef Chahine, "Gamila, al-Gaza'iriyya" [Yamila, la argelina] (1958).

${ }^{10}$ Dentro de este "paquete de medidas" podemos mencionar: el establecimiento del matrimonio civil, el voto universal femenino, el aumento de la edad mínima para el casamiento. Por otro lado, el gobierno colonial había lanzado una campaña el año anterior que buscaba realizar movilizaciones para que las argelinas se quitaran el velo como muestra de apoyo al gobierno francés. Finalmente, en el marco de la política de contrainsurgencia y guerra psicológica en el ámbito rural, el ejército francés
} 


\section{La construcción de una "nueva” mujer argelina: diario de una guerrillera}

del FLN en los años de guerra, no era sólo construir un Estado "moderno" e independiente en oposición a la imagen de retraso y dependencia del Estado colonial, sino que además buscaba construir de igual manera ciudadanos y ciudadanas acordes a los nuevos parámetros de modernidad y desarrollo que se vislumbraban en los discursos de esta fuerza política. Si bien la "originalidad de la identidad argelina" era algo recurrente en el discurso nacionalista, esta identidad pareció complementarse con la idea de un nacionalismo revolucionario que buscaba una transformación social para modernizar el país, y para que los argelinos y argelinas lograran desarrollar plenamente su identidad acorde a los nuevos vientos de época (Byrne, 2016). Finalmente, consideramos que privilegiar la voz de una mujer "moderna" en una columna del principal órgano de prensa del FLN -mujer joven, urbana y con acceso a estudios-, en lugar de elegir a una mujer de edad media, casada, con hijos/as y del ámbito rural -perfil de la mayor cantidad de mujeres que participaron de la guerra de independencia argelina- servía además como señal para demostrarle a un público internacional que la guerra y la independencia conducirían a la modernidad de Argelia.

En los discursos nacionalistas en general -y el argelino no escapó de esta lógica- las mujeres aparecieron como protectoras privilegiadas de la identidad nacional, de la tradición y las costumbres nacionales. En este proceso, la mujer era pensada como la última guardiana de la identidad nacional, ya que los efectos de la colonización habían provocado una atomización en la sociedad colonizada, convirtiendo a la familia en el último refugio para los valores y tradiciones que el sistema colonial había desestructurado (Yuval-Davis, 1997; Chaterjee, 1999; Jawavardena, 2016). Por este motivo, la subversión del rol de la mujer durante la guerra generó muchas ansiedades y tensiones en el movimiento nacionalista, por lo que el intento de querer mantener un equilibrio entre las posiciones más conservadoras y más modernizantes del movimiento no fue tarea fácil. En este contexto, es que se podría explicar el perfil de la joven elegida para escribir la columna: una joven que ya tenía permiso para salir de su casa a estudiar y no estaría rompiendo tantos tabúes al ausentarse de su hogar para participar de la lucha por la independencia.

En la primera entrega de "El Journal...", una vez inserta en la lucha política, la joven mujer que escribe la columna de "El Moudjahid" pareció vivir una síntesis de las experiencias adquiridas por muchas de las militantes urbanas: se ocupó del transporte de armas y medicamentos, fue arrestada, sufrió torturas -pero no denunció a sus compañeros- para luego ser liberada. En esta primera descripción, el discurso pareciera marcar el bautismo de fuego de todas las militantes para poder acceder al maquis: el martirio de la tortura y el pasaje a la clandestinidad. A su vez, la clandestinidad sería otra argumentación importante para justificar la salida de las jóvenes del hogar para unirse al maquis, ya que debido al riesgo que conllevaba estar en esa situación en la ciudad, era preferible aceptar la incorporación de las mujeres

había desarrollado grupos especiales (Équipes médico-sociales itinérants, EMSI), que tenían como objetivo entrar en contacto directo con mujeres locales como medio de desarrollar una guerra psicológica contra el FLN (MacMaster, 2009; Vince, 2015). 


\section{Julieta Chinchilla}

en el ámbito más masculino de la guerra. De hecho, la primera observación que hace la autora al llegar al maquis es señalar que es la única mujer en el lugar y que sólo hay 14 mujeres jóvenes en toda la wilaya ${ }^{11}$. Sin embargo, rápidamente señala la moral de las poblaciones cercanas, sobre todo la de las mujeres, que a pesar de ser el principal blanco de la represión, ni si quiera "se detienen a llorar a sus muertos" (El Moudjahid N45; 1959).

\section{Hacia una "nueva" mujer... bajo tutela}

Al final de la primera columna, la autora menciona a dos personajes que recorrerán las siguientes publicaciones y servirán como modelos a seguir. Por un lado, Malika, enfermera diplomada que supo luchar con arma en mano hasta su último suspiro para defender a sus pacientes, modelo para el resto de las combatientes por su entrega y heroísmo. La vida de Malika fue contada por el coronel Amirouche en una reunión con las mujeres del maquis y otras colaboradoras de las poblaciones cercanas (El Moudjahid $\mathrm{N}^{\circ} 44,1959$ ).

La figura de Amirouche también es resaltada en otras entregas. El coronel aparece como un referente, como aquel que le dio un espacio a las mujeres para participar en la guerra, el que les indica los modelos a seguir en ese nuevo contexto (como el ejemplo de heroísmo y entrega de Malika) y el que finalmente impulsa a la autora a escribir la columna para dar a conocer la experiencia de cientos de mujeres ${ }^{12}$. Si bien es cierto que, Amirouche fue uno de los pocos líderes del FLN que promovió la participación de las mujeres en el maquis, también es cierto que la mirada que tenía de sus compañeras no rompía del todo con la mirada más tradicional sobre el rol de la mujer. Amirouche, caracterizaba a las mujeres como "lo más puro de la revolución", por lo que las enviaba a organizar grupos de formación y atender al pueblo ya que "las mujeres son las que hacen mejor esta tarea" (El Moudjahid $\mathrm{N}^{\circ} 46$, 1959).

En resumen, desde la primera columna tenemos una figura masculina, fuente de autoridad para el resto de las mujeres, que permite mantener cierto equilibrio frente a la subversión que implica la presencia de mujeres en el maquis-, ya que la figura de este hombre pareciera representar la de un padre que ofrece protección a las mujeres y cuida de ellas otorgándoles actividades que no rompan de una manera tajante con los roles tradicionales que les fueron conferidos, es decir, actividades de cuidado y educativas.

\footnotetext{
${ }^{11}$ El término wilaya se utilizó durante la guerra para señalar las unidades territoriales o regiones militares del ALN/FLN. En total, el territorio argelino, fue dividido en 6 wilayas, más la zona autónoma de Argel. Posteriormente, al llevar la guerra a la metrópoli, se sumó la wilaya 7 correspondiente a Francia. Actualmente el país cuenta con 58 wilayas o departamentos.

${ }^{12}$ La primera columna de "Journal d'une maquisarde" aparece tres meses después de la muerte del coronel Amirouche y luego de que la mayoría de las militantes fueran evacuadas a las fronteras de Túnez y Marruecos como consecuencia del aumento de la represión.
} 


\section{La construcción de una "nueva" mujer argelina: diario de una guerrillera}

Además de contar, en cada entrega, diferentes experiencias personales y de otras mujeres en el maquis, los relatos que más abundan son los de heroísmo. A pesar que la autora señala que prefiere que las llamen "simplemente argelinas" en vez de heroínas, porque lo que intenta hacer es testimoniar sobre lo que es y será la argelina en un país independiente, lo cierto es que a través de este tipo de relatos se busca legitimar el rol de la mujer en la futura Argelia independiente (El Moudjahid $\mathrm{N}^{\circ} 48,1959$ ). Por otro lado, en estos relatos donde se destaca la entrega de las mujeres sin buscar ningún reconocimiento a cambio, se resalta que su principal objetivo es mantenerse cerca de sus "hermanos" (El Moudjahid $\mathrm{N}^{\circ} 45,1959$ ). En otras palabras, en diversos momentos del relato de la maquisarde, se puede observar que la figura de la mujer aparece siempre bajo la protección de una figura masculina o la justificación de la presencia de las mujeres en espacios que no son los tradicionales para ellas, es para estar cerca de los hombres y "ayudarlos".

Otro punto interesante de estas crónicas, es el hecho que la autora menciona que los jefes reunían a las militantes en cada ocasión que podían para que pudieran debatir sobre "el rol de la mujer en la lucha por la liberación nacional" (El Moudjahid $\mathrm{N}^{\circ} 45$, 1959). Este fue el caso de la reunión que se realizó en 1957 en Palestro, una de las reuniones más importantes que recuerda la columnista, a pesar que ninguna militante había pedido esa reunión expresamente. Allí, la autora nos cuenta que pudo encontrarse con mujeres de todo el país y de todas las extracciones sociales, en donde pudieron intercambiar experiencias. Resulta interesante observar que al momento de relatar esta reunión, la autora hace hincapié sobre los elementos que impulsaron a las mujeres a entrar en la lucha por la independencia. Allí todas coincidieron en que decidieron pasar a la acción al tomar conciencia de las injusticias del colonialismo y de la opresión extranjera. Es decir, nada de lo que impulsó la participación femenina en la guerra de independencia se relacionaba a su situación en tanto mujeres dentro de la sociedad colonial.

\section{Conclusiones}

A partir de los puntos señalados de la columna "Journal d'une maquisarde", podríamos concluir, en primer lugar, que los relatos de heroísmo y entrega de las mujeres reseñadas buscan darle legitimidad a la participación de las mujeres en la lucha por la independencia de Argelia, en un contexto en el que la presencia de mujeres en el maquis u otras células revolucionarias en la ciudad generaban tensiones y ansiedades. Por otro lado, el hecho de que el diario de comunicación oficial del FLN fuera el que le diera ese espacio a la participación de mujeres, construía legitimidad para la formulación de un nuevo estatus de las mismas en la etapa independiente (Chaulet, 2012). El heroísmo -como característica generalizada de todas las combatientes-, no entraría en contradicción con la idea de "mujer guardiana de la identidad nacional", ya que las combatientes, luchando por la 


\section{Julieta Chinchilla}

independencia y cuidando a sus "hermanos", estaban llevando hasta las últimas consecuencias ese mandato de género.

Si bien la columna fue escrita desde la frontera tunecina con Argelia -como se mencionó-, la publicación da cuenta de los cambios de valores que se dieron en cuanto al rol de la mujer en la sociedad argelina durante la guerra, no sin tensiones. Por un lado, se buscó la voz en primera persona de una mujer joven urbana con estudios, para evitar romper por un lado con tabúes impuestos a las mujeres del ámbito rural que hubieran sido muy difíciles de ser aceptados por el conjunto del movimiento que integraba las filas del FLN. Por otro lado, el perfil de la columnista también responde a la idea de una mujer/Argelia moderna que se busca construir tras la obtención de la independencia. Por último, el hecho mismo de darle un espacio a la mujer en el principal órgano de comunicación del FLN, también respondería a una manera de presentar al FLN como una fuerza política modernizadora, en consonancia con la mirada que también describía uno de los principales intelectuales de la revolución argelina, Frantz Fanon. Este autor, en su artículo "Año V de la Revolución", desarrolló una visión optimista sobre el rol de la mujer en la nueva sociedad a partir de señalar la masiva participación de las mujeres en la guerra y analizar la rápida capacidad que tuvieron en adaptare al nuevo contexto y enfrentar los diferentes peligros (Fanon, 1971).

Finalmente, este lugar privilegiado de la mujer como forma de destacar el objetivo modernizante de la futura Argelia (sobre todo de cara a la opinión pública internacional), no debe dejar escapar que no se hizo sin tensiones ni resguardos. En primer lugar, no habría que dejar de tener en cuenta que las columnas fueron editadas bajo la supervisión masculina de los "jefes". También es importante subrayar que la ruptura que implicaba la participación de las mujeres en la guerra de independencia se trató de moderar a través del discurso. La referencia constante a los "jefes" como modelos a seguir -particularmente la figura del coronel Amirouche- pero también como protectores y guardianes de las mujeres es constante a lo largo de las entregas del "Journal...".

En síntesis, a través de esta columna se pueden rastrear los cambios de valores en cuanto a las relaciones de género en el corto plazo, y a partir de ello vislumbrar la génesis de una nueva subjetividad femenina y política. En este testimonio podemos ver cómo este conflicto impulsó la participación de las mujeres en la política y en la guerra, rompiendo con tradiciones que las retenían dentro del ámbito de su hogar, además de crear espacios mixtos.

También es importante tener en cuenta que dentro de esta columna existen todos los elementos que permanecerán en el discurso nacionalista tras la independencia. Vemos cómo el modelo de mujer-heroína-mártir es el que se construye a lo largo de las entregas, donde además se privilegia la voz de las mujeres que combatieron -o estuvieron cerca de los combatientes-, por sobre las militantes civiles (moussebilines) que fueron mayoría dentro del movimiento. Estos cambios en la construcción de nuevas subjetividades no se dieron sin tensiones, por lo que dentro del testimonio se buscó equilibrar ciertas innovaciones con costumbres arraigadas 
sobre el rol de la mujer. En primer lugar, se eligió a una joven citadina que ya tenía permiso de salir de su hogar para ir a estudiar, intentando dar cuenta que la militancia de las mujeres urbanas se dio de "una manera más natural" (El Moudjahid $\left.\mathrm{N}^{\circ} 45,1959\right)$, por lo que no rompería con tantos tabúes. También se insistió a lo largo de las columnas en la presencia de figuras masculinas, con autoridad, fuerte moral y con características paternalistas que recrearan la dinámica de un hogar, que a su vez sirvió para atenuar un tipo de relación socialmente perjudicable (la convivencia entre hombre y mujeres) y traducirla con este lenguaje como una relación familiar, y por lo tanto aceptable.

\section{Bibliografía}

AAVV (2011). El Moudjahid. Un journal de combat (1956-1962). Rouiba: Éditions ANEP.

Amrane-Minne, D. (1994). Des Femmes Dans la Guerre D’Algérie. Condé-SurNoireau : Karthala.

Byrne, J (2016). Mecca of Revolution. Algeria, decolonization and the Third World order. New York: Oxford University Press.

Capdevila, L. (2011). Identités de genre et événement guerrier. Des expériences féminines du combat. Sextant/Femmes en guerre, (28).

Chaterjee, P. (1999). La nación y sus mujeres. En S. Dube (coord.) Pasados poscoloniales. Colección de ensayos sobre la historia y la etnografía de la India. CMX: El Colegio de México.

Chaulet Achour, C. (2012). Écrits d'algériennes et guerre d'indépendance. Témoignages et créations. CAIRN, (81).

Chinchilla, J. (2014). La mujer argelina como elemento de negociación en la construcción de un nuevo poder político: de la independencia a la crisis del sistema de partido único. Relaciones Internacionales, (27).

Fanon, F. (1971). Dialéctica de la Liberación. Montevideo: Editorial Cienfuegos.

Halimi, G y Beauvoir, S. (1964). Djamila Boupacha, proceso a la tortura. Barcelona: Seix Barral.

Harbi, M. y Stora, B. (2012). La guerre d'Algérie. Millau : Pluriel.

Lacheraf, M. (1967). Argelia: Nación y Sociedad. La Habana: Instituto del Libro. 


\section{Julieta Chinchilla}

Lalami, F. (2008) L'enjeu du statut des femmes durant la période coloniale en Algérie. Nouvelles Questions Féministes, 27, (3).

MacMaster, N. (2009). Burning the veil. The Algerian war and the "emancipation" of Muslim women, 1954-1962. Manchester : Manchester University Press.

Meynier G. (2002). Histoire intérieure du FLN. 1954-1962. Paris: Fayard.

Qasim A. (2000). The Liberation of Women: Two Documents in the History of Egyptian Feminism. El Cairo : AUC Press.

Sambron, D. (2009). Femmes Musulmanes. Guerre d'Algérie 1954-1962. Paris: Édition Autrement.

Vince, N. (2015). Our fighting sisters. Nation, memory and gender in Algeria, 19542012. Manchester: Manchester University Press.

Yuval-Davis, N. (1997). Gender and Nation. London: SAGE Publications.

\section{Fuentes}

El Moudjahid N 44, 22/06/1959, p. 2.

El Moudjahid $\mathrm{N}^{\circ} 45,02 / 07 / 1959$, p. 9.

El Moudjahid $\mathrm{N}^{\circ} 46,28 / 07 / 1959$, p. 7.

El Moudjahid No 47, 03/08/1959, p. 12.

El Moudjahid No 48, 17/08/1959, p. 10.

El Moudjahid № 49, 31/08/1959, p. 12.

Recibido: 30/11/2020

Evaluado: 20/01/2021

Versión Final: 26/02/2021 